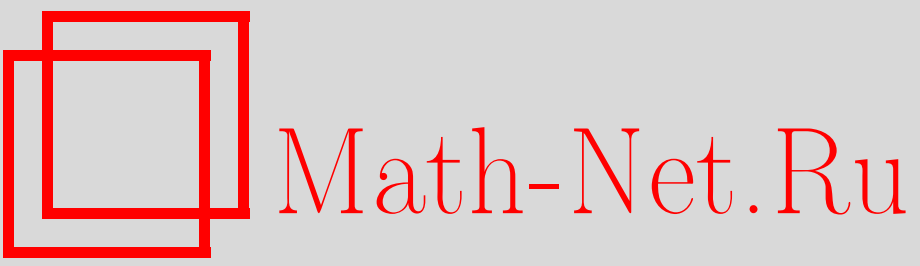

Б. Г. Конопельченко, А. Моро, Описание распространения света в нелинейной среде Коул-Коула с помощью уравнения Бюргерса-Хопфа, ТМФ, 2005, том 144, номер 1, 102-109

DOI: https://doi.org/10.4213/tmf1836

Использование Общероссийского математического портала Math-Net.Ru подразумевает, что вы прочитали и согласны с пользовательским соглашением

http: //www . mathnet.ru/rus/agreement

Параметры загрузки:

IP: 54.172 .240 .79

26 апреля 2023 г., 12:35:52 
ТЕОРЕТИЧЕСКАЯ

И МАТЕМАТИЧЕСКАЯ

ФИЗИКА

Том 144, № 1

июль, 2005

(C) 2005 г.

Б. Г. Конопельченко*, А. Моро*

\section{ОПИСАНИЕ РАСПРОСТРАНЕНИЯ СВЕТА В НЕЛИНЕЙНОЙ СРЕДЕ КОУЛ-КОУЛА С ПОМОЩЬЮ УРАВНЕНИЯ БЮРГЕРСА-ХОПФА}

Недавно была предложена новая модель распространения света в так называемой слабо нелинейной трехмерной среде Коул-Коула с нелокальностью малого радиуса действия. Было показано, что в пределе геометрической оптики эта модель является интегрируемой и подчиняется бездисперсионному уравнению Веселова-Новикова, $(1+1)$-мерная редукция которого приводит к уравнению Бюргерса-Хопфа. Свойства последнего обсуждаются в контексте нелинейной геометрической оптики. В качестве иллюстрации рассмотрен явный пример.

Ключевые слова: нелинейная оптика, интегрируемые системы.

Многочисленные современные исследования, касающиеся бездисперсионных интегрируемых систем, продемонстрировали значимость таких систем для широкого круга областей физики, таких как лапласовский рост, топологическая теория поля, нелинейная оптика [1]-[3], а также их глубокую связь с различными областями математики, в частности, с теорией асимптотических разложений, конформными и квазиконформными отображениями и исследованием интегрируемых деформаций комплексных алгебраических кривых [4].

В настоящей работе нас интересует применение теории бездисперсионных интегрируемых систем в нелинейной геометрической оптике. В частности, в работе [2] было показано, что уравнения Максвелла, описываюшие распространение света в слабо нелинейной трехмерной среде Коул-Коула, интегрируемы в пределе геометрической оптики. В этом пределе система подчиняется бездисперсионной иерархии Веселова-Новикова $(\mathrm{BH})$, к которой применим квазиклассический метод $\bar{\partial}$-одевания [3].

Основанный на симметрийных ограничениях метод редукции [5] представляется эффективным способом вычисления решений бездисперсионного уравнения ВН [6].

В дальнейшем мы сконцентрируем внимание на $(1+1)$-мерной редукции для случая, когда показатель преломления не зависит от одной координаты. При этом бездисперсионная иерархия ВН сводится к так называемой иерархии Бюргерса-Хопфа (БХ). Для

* Dipartimento di Fisica dell'Università di Lecce and INFN, Sezione di Lecce, I-73100 Lecce, Italy. E-mail: Boris.Konopeltchenko@le.infn.it, antonio.moro@le.infn.it 
простоты мы будем рассматривать первые уравнения иерархий, именуемые бездисперсионным уравнением ВН и уравнением БХ.

В этом отношении определенный интерес представляет интерпретация некоторых свойств уравнения БХ применительно к случаю нелинейной геометрической оптики. Одно из таких свойств связано с сушествованием опрокидьваюшихся волновых решений. Эти решения могут быть полезны для моделирования диэлектриков, которые содержат своего рода "примеси", вызывающие резкие изменения показателя преломления. Действительно, можно видеть, что в так называемых точках опрокидывания кривизна световых лучей претерпевает разрыв, подобно тому, как это имеет место на гранище раздела различных сред.

Начнем с уравнений Максвелла в диэлектрической среде

$$
\begin{array}{ll}
\nabla \wedge \mathbf{H}-\frac{\partial \mathbf{D}}{\partial t}=0, & \nabla \cdot \mathbf{D}=0, \\
\nabla \wedge \mathbf{E}+\frac{\partial \mathbf{B}}{\partial t}=0, & \nabla \cdot \mathbf{B}=0,
\end{array}
$$

рассматриваемых вместе с материальными уравнениями

$$
\mathbf{D}=\varepsilon \mathbf{E}, \quad \mathbf{B}=\mu \mathbf{H} .
$$

Будем искать монохроматические решения вида

$$
\begin{aligned}
\mathbf{E}(x, y, z, t) & =\mathbf{E}_{0}(x, y, z) e^{-i \omega t}, \\
\mathbf{H}(x, y, z, t) & =\mathbf{H}_{0}(x, y, z) e^{-i \omega t} .
\end{aligned}
$$

Тогда уравнения (1), (2) приводят к хорошо известным уравнениям второго порядка:

$$
\begin{aligned}
\nabla^{2} \mathbf{E}_{0}+\omega^{2} \mu \varepsilon \mathbf{E}_{0}+(\nabla \ln \mu) \wedge\left(\nabla \wedge \mathbf{E}_{0}\right)+\nabla\left(\mathbf{E}_{0} \cdot \nabla \ln \varepsilon\right) & =0 \\
\nabla^{2} \mathbf{H}_{0}+\omega^{2} \mu \varepsilon \mathbf{H}_{0}+(\nabla \ln \varepsilon) \wedge\left(\nabla \wedge \mathbf{H}_{0}\right)+\nabla\left(\mathbf{H}_{0} \cdot \nabla \ln \mu\right) & =0 .
\end{aligned}
$$

Исследуемая среда характеризуется следуюшими свойствами [2], [3].

1. Величины $\varepsilon$ и $\mu$ подчиняются закону дисперсии Коул-Коула

$$
\begin{aligned}
\varepsilon & =\varepsilon_{0}+\frac{\tilde{\varepsilon}}{1+\left(i \omega \tau_{0}\right)^{2 \nu}}, \quad 0<\nu<\frac{1}{2} . \\
\mu & =\mu_{0}+\frac{\tilde{\mu}}{1+\left(i \omega \tau_{0}\right)^{2 \nu}},
\end{aligned}
$$

Подчеркнем, что диапазон значений показателя степени $\nu$ играет ключевую роль при построении интегрируемого предела высоких частот.

2. Величины $\varepsilon_{0}$ и $\mu_{0}$ зависят только от координат $x, y$ и $z$, в то время как величины $\tilde{\varepsilon}$ и $\tilde{\mu}$ предполагаются зависимыми от координат, полей и пространственных производных от полей. Последнее обстоятельство может быть объяснено на основе механизма нелокальности малого радиуса действия с помошью интегрального материального уравнения, связываюшего напряженность электрического поля $\mathbf{E}$ и вектор электрической индукции $\mathbf{D}[3]$. 
3. В пределе высоких частот все величины демонстрируют медленную зависимость от переменной $z$, что формально можно записать следующим образом:

$$
\frac{\partial}{\partial z}=\omega^{-\nu} \frac{\partial}{\partial \xi}
$$

где $\xi$ - "медленная" переменная, определяемая формулой $z=\omega^{\nu} \xi$. Более того, любая функция $f(x, y, z)$ может быть разложена по степеням параметра $\omega^{\nu}$ в асимптотический ряд вида

$$
f(x, y, z)=f(x, y, \xi)+\omega^{-\nu} f_{1}(x, y, \xi)+\omega^{-2 \nu} f_{2}(x, y, \xi)+\cdots
$$

Представляя $\mathbf{E}_{0}$ и $\mathbf{H}_{0}$ как

$$
\mathbf{E}_{0}=\widetilde{\mathbf{E}}_{0} e^{i \omega S}, \quad \mathbf{H}_{0}=\widetilde{\mathbf{H}}_{0} e^{i \omega S}
$$

и используя приведенные выше предположения в одном из уравнений (4), в пределе высоких частот $(\omega \rightarrow \infty)$ получаем в порядках $\omega^{2}$ и $\omega^{2-2 \nu}$ главные нетривиальные вклады $[2],[3]$

$$
\begin{gathered}
S_{x}^{2}+S_{y}^{2}=4 u, \\
S_{\xi}=\varphi\left(x, y, \xi, S_{x}, S_{y}\right) .
\end{gathered}
$$

Уравнение (7) - обычное уравнение эйконала в двумерном пространстве, $\varphi$ - некоторая функция, $4 u=\varepsilon_{0} \mu_{0}$. Как обсуждалось в работе [3], уравнения (7) и (8) образуют переопределенную систему для фазы $S$. Условие совместности налагает определенные ограничения на возможный вид функции $\varphi$ и допустимые показатели преломления $u$. При полиномиальной зависимости $\varphi$ от производных фазы $S$ первый нетривиальный случай отвечает полиному третей степени:

$$
\varphi=\frac{1}{4} S_{x}^{3}-\frac{3}{4} S_{x} S_{y}^{2}+V_{1} S_{x}+V_{2} S_{y}
$$

Условие совместности дает уравнения

$$
\begin{gathered}
u_{\xi}=\left(V_{1} u\right)_{x}+\left(V_{2} u\right)_{y} \\
V_{1 x}-V_{2 y}=-3 u_{x} \\
V_{2 x}+V_{1 y}=3 u_{y}
\end{gathered}
$$

которые суть бездисперсионное уравнение ВН. В работе [6] было показано, как симметрийные ограничения позволяют построить его $(1+1)$-мерные редукции гидродинамического типа. В работе [3] был рассмотрен ряд явных решений комплексного бездисперсионного уравнения ВН.

Частньй случай, при котором показатель преломления зависит только от одной координаты в $x y$-плоскости, также представляет физический интерес. Предположим, таким образом, что

$$
u_{y}=0
$$


Из уравнения эйконала (7) следует, что фазовая функция $S$ должна удовлетворять условию $S_{y}=c, c=$ const; тогда

$$
S=c y+\widetilde{S}(x, \xi),
$$

где $\widetilde{S}$ не зависит от $y$. Уравнение эйконала (7) принимает вид

$$
\widetilde{S}_{x}^{2}=4 u-c^{2}
$$

в то же время, выбирая $V_{2}=0$, получаем

$$
V_{1}=-3 u
$$

и бездисперсионное уравнение ВН (10) сводится к уравнению БХ

$$
u_{\xi}+6 u u_{x}=0 .
$$

Решения уравнения БХ могут быть выражены в форме соотношений годографа следующим образом [7]:

$$
x-6 u \xi+\psi(u)=0,
$$

где $\psi$ - произвольная функция своего аргумента. Более того, как только функция $u(x, \xi)$ найдена из уравнения (15), фазовая функция выписывается явно:

$$
S=c y \pm \int \sqrt{4 u-c^{2}} d x .
$$

Сингулярная структура уравнения БХ связана с существованием точки опрокидывания $\xi^{*}$, в которой имеет место условие

$$
u_{x} \rightarrow \infty .
$$

В теории дифференциальных уравнений в частных производных такие решения называются опрокидывающимися волнами. Вычисление точки опрокидывания является учебным упражнением [7]. Например, выбирая начальное условие в виде $u(x, 0)=(1-$ th $x) / 6$, имеем $\xi^{*}=1$ (рис. 1 ).

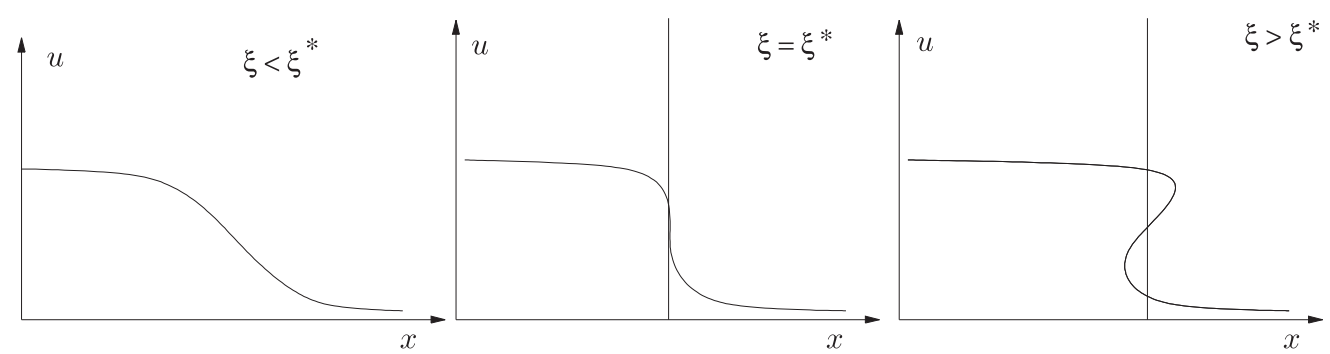

Рис. 1. Типичное опрокидывающееся решение уравнения БХ. За точкой опрокидывания $\left(\xi>\xi^{*}\right)$ функция $u$ становится многозначной. 


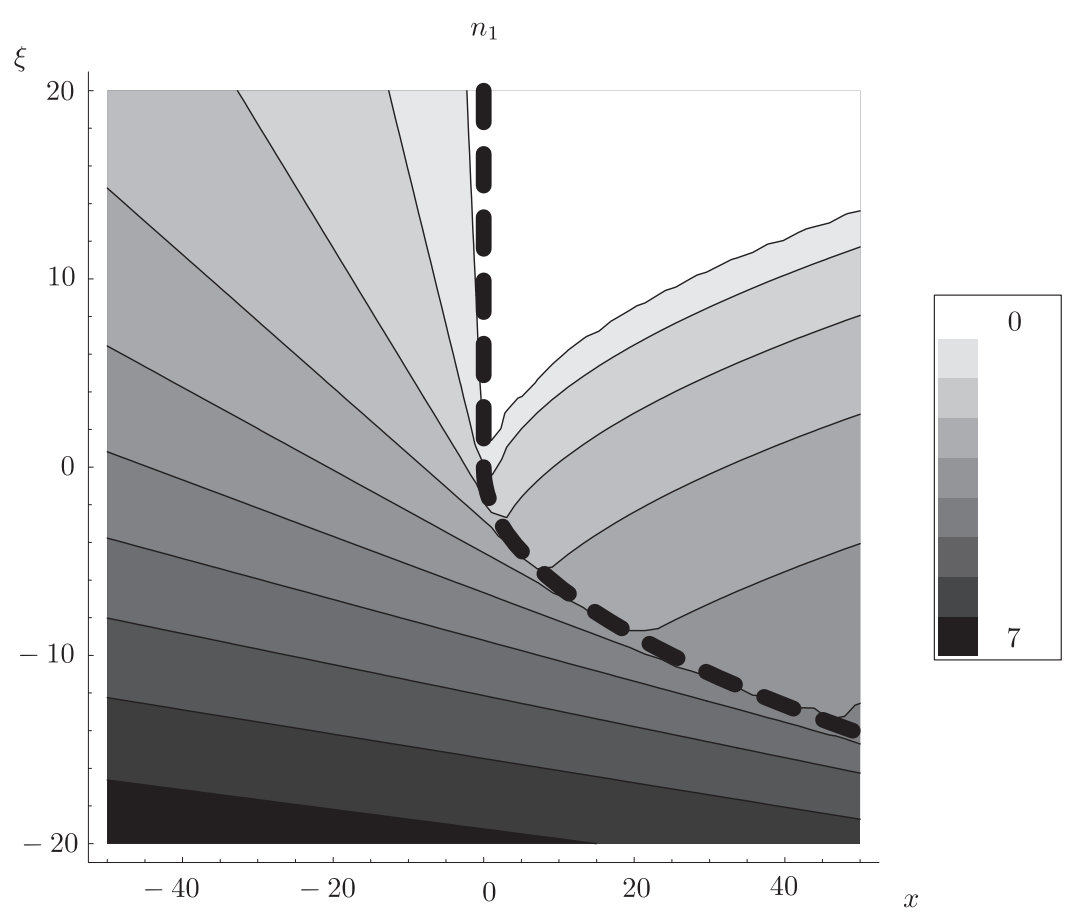

Рис. 2. График плотности вещественной части показателя преломления $n=n_{1}+i n_{2}$. Штриховая линия определяет границу области с ненулевой мнимой частью показателя преломления.

Отметим, что, как обсуждалось в работе [8], сингулярный сектор иерархии БХ вызывает расслоение аффинного пространства независимых переменных, которое дает начало интегрируемым деформациям гиперэллиптических кривых.

Распространение света на плоскости $\pi: \xi=\xi_{0}$ ( $\xi_{0}=$ const) подчиняется обычному уравнению эйконала (7). Обозначив через $\Sigma$ конгруэнцию на $\pi$ перпендикулярно семейству кривых $S\left(x, y, \xi_{0}\right)=\lambda$, параметризованному при помощи $\lambda$, можно представить кривизну кривой $\gamma \in \Sigma$ известной формулой [9]

$$
\kappa=\frac{1}{2 u} \nu \cdot \nabla u,
$$

где $\nabla=\left(\partial_{x}, \partial_{y}\right)$, а $\boldsymbol{\nu}=\left(\nu_{1}, \nu_{2}\right)$ - единичная главная нормаль к $\gamma$ на плоскости $\pi$. Заметим теперь, что кривая $\gamma$ локально совпадает с проекцией светового луча на плоскость $\pi$.

В силу условия (11) формула (18) принимает вид

$$
\kappa=\frac{\nu_{1}}{2 u} u_{x}
$$

Далее, на основании того, что величина $u_{x}$ претерпевает разрыв, мы заключаем, что кривизна светового луча также претерпевает разрьв. Это типичное поведение световых лучей на границе раздела различных сред. Решения такого рода могут быть полезны для описания ситуаций, в которых световые лучи распространяются в неоднородной 

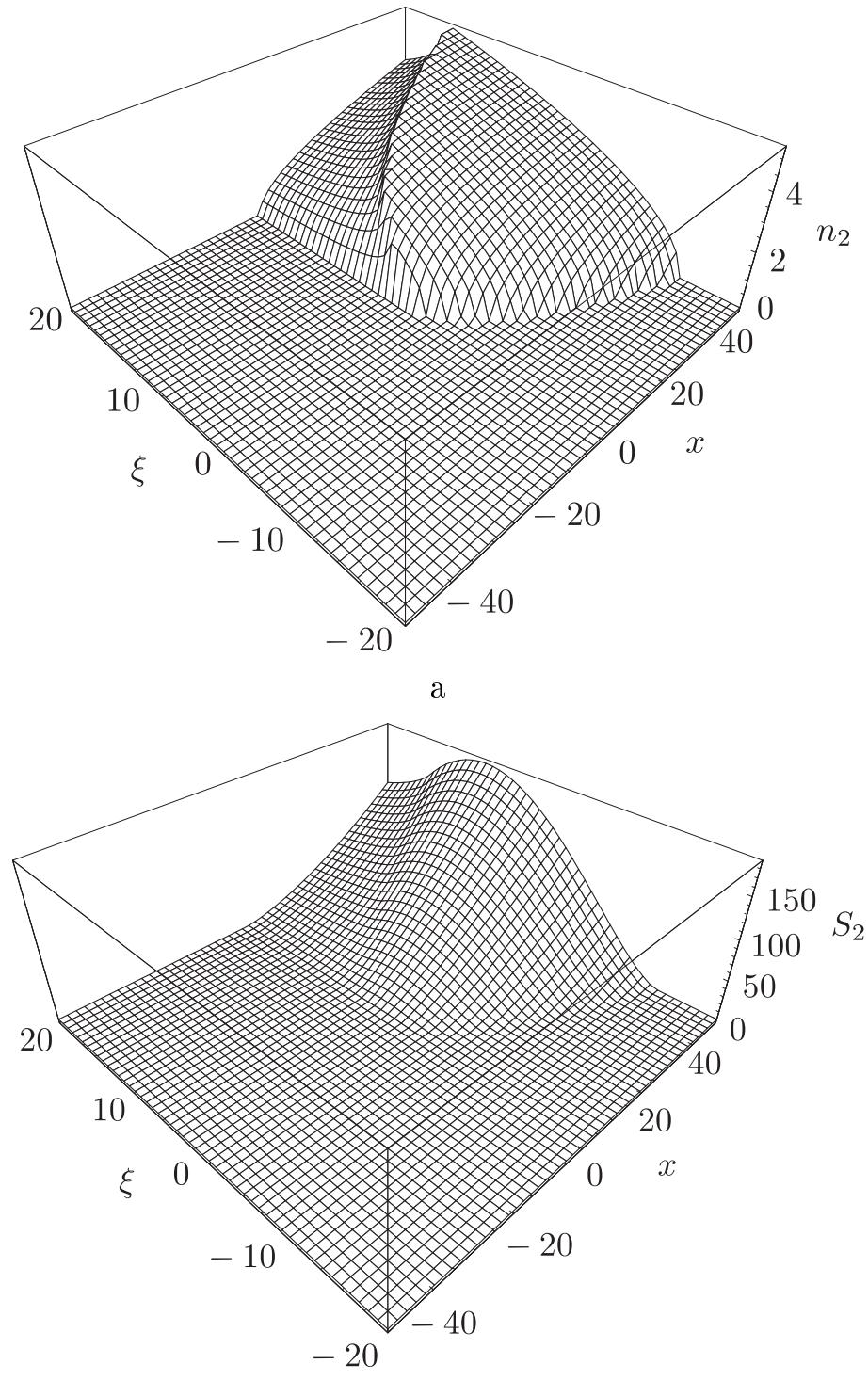

б

Рис. 3. а) Трехмерный график мнимой части показателя преломления. б) Трехмерный график мнимой части фазы $S$. Мнимая часть обрашается в нуль везде, за исключением области поглощения.

среде, встречая в определенных точках на своем пути те или иные примеси. Эти примеси, оптические свойства которых радикально отличаются от свойств остальной среды, могут обуславливать резкое изменение направления распространения.

Как хорошо известно из электродинамики, комплексные показатели преломления тоже имеют физический смысл, поскольку могут быть использованы для описания эффектов поглошения излучения. В нашем конкретном случае комплексные значения показа- 


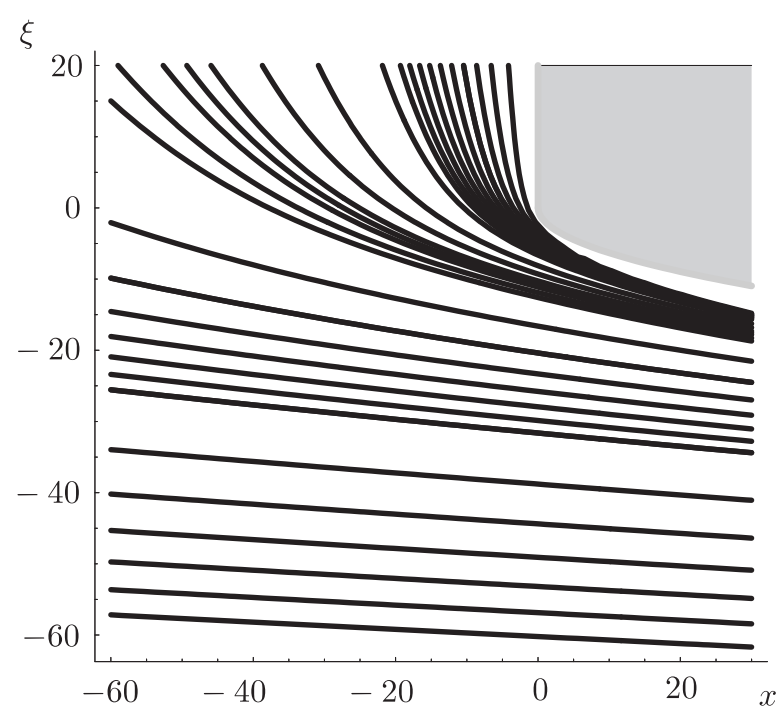

Рис. 4. Конфигурация волновых фронтов на плоскости $(x, \xi)$.

теля преломления $n$ соответствуют сильному затуханию электромагнитных волн. Этот факт мы продемонстрируем на следующем явном примере.

Рассмотрим решение, получаюшееся из соотношения годографа (15), если положить в нем $\psi(u)=u^{2}$. Явно выражая $u$ из $(15)$ и подставляя в выражение $(16)$, получаем

$$
\begin{aligned}
u & =\frac{1}{2}\left(-6 \xi+\sqrt{36 \xi^{2}-4 x}\right), \\
S & =\frac{4}{15} \sqrt{2} \sqrt{-6 \xi+\sqrt{36 \xi^{2}-4 x}}\left(6 \xi\left(-6 \xi+\sqrt{36 \xi^{2}-4 x}\right)+12 x\right) .
\end{aligned}
$$

Заметим, что показатель преломления $n=2 \sqrt{u}$ (мы не рассматриваем отрицательные показатели преломления) не является вешественным во всей $x \xi$-плоскости. В тех областях, где коэффициент преломления принимает комплексные значения, фазовая функция $S$ также становится комплекснозначной. Таким образом, полагая $S=S_{1}+i S_{2}$, мы видим, что если $S_{2}>0$, то электрическое поле приобретает экспоненциально затухающий множитель

$$
\mathbf{E}=\mathbf{E}_{0} e^{-\omega S_{2}} e^{i \omega S_{1}}
$$

В этих областях имеют место сильные эффекты поглошения.

На рис. 2 представлена зависимость вешественной части показателя преломления $n=2 \sqrt{u}$ от координаты $x$.

На рис. За приведен трехмерный график зависимости мнимой части показателя преломления от $x, t$. Там, где показатель преломления комплексный, мнимая часть фазы $S$ также отлична от нуля, что указывает на сильное поглощение (см. рис. 3б).

На рис. 4 представлена конфигурация волновых фронтов на $x \xi$-плоскости. Затененная область представляет область поглошения. При $\xi \rightarrow-\infty$ мы имеем почти плоский 
волновой фронт. По направлению к области поглошения волновые фронты деформируются, следуя линиям уровня показателя преломления. В непосредственной близости от области поглощения волновые фронты отклоняются почти под прямым углом.

Благодарности. Работа частично поддержана COFIN PRIN “SINTESI” 2002

\section{Список литературы}

[1] I. K. Kostov, I. Krichever, M. Mineev-Weinstein, P. B. Wiegmann, A. V. Zabrodin. The $\tau$-function for analytic curves. In: Random Matrix Models and Their Applications. Math. Sci. Res. Inst. Publ. V. 40. Eds. P. Bleher, A. Its. Cambridge: Cambridge Univ. Press, 2001. P. 285-299; M. Mineev-Weinstein, P. B. Wiegmann, A. V. Zabrodin. Phys. Rev. Lett. 2000. V. 84. № 22. P. 5106-5109; P. B. Wiegmann, A. V. Zabrodin. Commun. Math. Phys. 2000. V. 213. P. 523-538; I. Krichever, M. Mineev-Weinstein, P. B. Wiegmann, A. V. Zabrodin. Physica D. 2004. V. 198. P. 1-28; nlin.SI/0311005; Commun. Math. Phys. 1992. V. 143. P. 415-429; Commun. Pure Appl. Math. 1994. V. 47. P. 437-475; B. A. Dubrovin. Commun. Math. Phys. 1992. V. 145. P. 195-207; Nucl. Phys. B. 1992. V. 379. P. 627-689.

[2] B. G. Konopelchenko, A. Moro. J. Phys. A. 2004. V. 37. P. L105-L111.

[3] B. G. Kononopelchenko, A. Moro. Stud. Appl. Math. 2004. V. 113. P. 325-352; nlin.SI/0403051.

[4] P. D. Lax, C. D. Levermore. Commun. Pure Appl. Math. 1983. V. 36. P. 253-290; P. 571-593; P. 809-830; B. G. Konopelchenko, L. Martinez Alonso. Phys. Lett. A. 2001. V. 286. P. 161-166; B. G. Konopelchenko, L. Martinez Alonso. J. Math. Phys. 2002. V. 43. № 7. P. 3807-3823; J. Phys. A. 2004. V. 37. P. 7859-7877; nlin.SI/0403052.

[5] L. Bogdanov, B. G. Konopelchenko. Phys. Lett. A. 2004. V.330. P.448-459; nlin.SI/0312013.SI.

[6] Л. В. Богданов, Б. Г. Конопельченко, А. Моро. Фунд. прикл. матем. 2004. Т. 10. С. 5-15; nlin.SI/0406023.

[7] Джс. Уизем. Линейные и нелинейные волны. М.: Мир, 1977.

[8] Y. Kodama, B. G. Konopelchenko. J. Phys. A. 2002. V. 35. P. L489-L500.

[9] M. Born, E. Wolf. Principles of Optics. Oxford: Pergamon Press, 1980. 